\title{
Human skull shape and masticatory induced stress: Objective comparison through the use of non-rigid registration
}

\author{
G. J. Jansen van Rensburg ${ }^{1,2}$, S. Kok ${ }^{1}$, D. N. Wilke ${ }^{2}$ \\ ${ }^{1}$ Modelling and Digital Science, The Council for Scientific and Industrial Research, South Africa \\ (jjvrensburg@csir.co.za) \\ ${ }^{2}$ Department of Mechanical and Aeronautical Engineering, University of Pretoria
}

\section{Summary}

Variation in masticatory induced stress, caused by shape changes in the human skull, is quantified in this article. A comparison on masticatory induced stress is presented subject to a variation in human skull shape. Non-rigid registration is employed to obtain appropriate computational domain representations. This procedure allows the isolation of shape from other variations that could affect the results. An added benefit, revealed through the use of non-rigid registration to acquire appropriate domain representation, is the possibility of direct and objective comparison and manipulation. The effect of mapping uncertainty on the direct comparison is also quantified. As shown in this study, exact difference values are not necessarily obtained, but a non-rigid map between subject shapes and numerical results gives an objective indication on the location of differences.

\section{Introduction}

The study of functional morphology considers the relationship between form and function. Evolutionary biologists, palaeontologists and anthropologists use numerical tools to enquire into the adaptation of organic form to accommodate the relevant physics [1, 2].

This article aims to compare masticatory induced stress fields subject to skull shape. Two human skull geometries are available for this comparison. The effect of skull shape on the masticatory induced stress field is inspected to assess the feasibility of performing a larger study on the functional morphology of the human skull. Two options are considered and illustrated in this article to compare the stress fields resulting from finite element analysis (FEA) [3].

The first option uses separate analyses on independently generated meshes. Each mesh is obtained from a digitally reconstructed skull geometry. The stress fields are then visually compared. This qualitative comparison could be influenced by observer bias and experience as well as unwanted or indeterminate variation in the analysed computational domains.

The second option uses approximate computational domain representations of the two different skull geometries, obtained through elastic or non-rigid registration. This is achieved by an approach that deforms a chosen generic mesh (also called the base mesh, deformable mesh or model shape) into a shape that resembles a different but related geometry. An unintended benefit of computational domains obtained through the use of non-rigid registration is a retrievable 


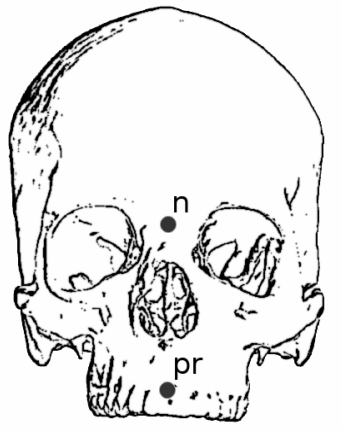

(a)

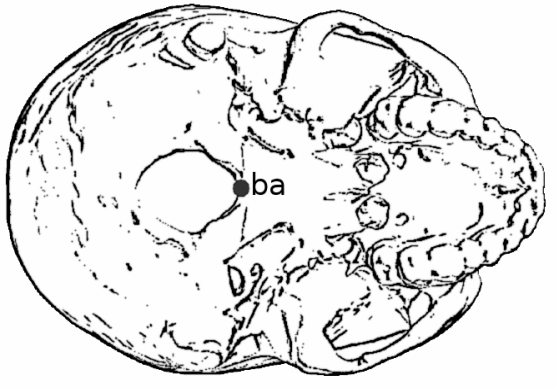

(b)

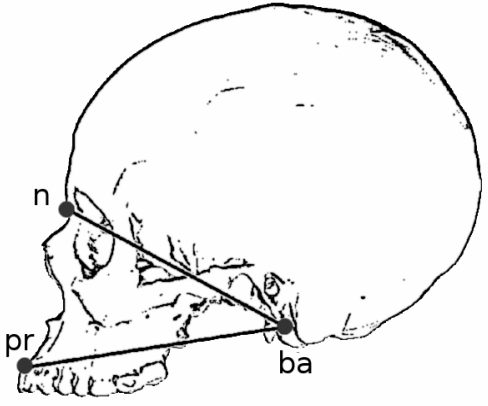

(c)

Figure 1: Landmarks on the (a) front, (b) side and (c) bottom of the human skull.

one to one map between these domains. A direct and objective comparison is possible because of this mapping.

A brief introduction to the intended study is presented. Section 2 covers aspects of independent domain analyses. In Section 3, the use of non-rigid registration is motivated, and the registration procedure used in this study is introduced. A direct and objective stress field comparison is then presented in Section 4 on the bite-induced stress results, using representative domains obtained through constrained non-rigid registration. In this study, only the effect of skull shape on the stress field is considered. The effects of material property and topological variation are deliberately excluded.

\subsection{Context of the study used}

The feasibility of a study on the effect of prognathism on masticatory induced stress in the human skull is inspected. Maxillary alveolar prognathism is defined as the percentage relationship between the distances of two lines. The origin of both lines are at the cranial base (ba) and through the cranial landmark positions of nasion (n) and prosthion (pr). This attribute is characterised by either one or both jaws projecting forward, and it influences the general shape of the maxillofacial region of the skeleton. The locations of these landmarks are visible in Figure 1.

Expressed as a percentage quantity, the distance ratio is termed the alveolar or gnathic index (GI) with

$$
\mathrm{GI}=\frac{\|\mathrm{pr}-\mathrm{ba}\|}{\|\mathrm{n}-\mathrm{ba}\|} \times 100,
$$

where $\|\bullet\|$ indicates the $l^{2}$-norm.

Skulls with a gnathic index below 97.9 are orthognathous. Mesognathous skulls have an index between 98 and 102.9 while prognathous skulls have a GI value above 103 [4].

In this paper, a stress field comparison is performed on a prognathic $(\mathrm{GI}=106.9)$ and orthognathic skull $(\mathrm{GI}=91.5)$.

\section{Independent mesh generation and analysis}

The first option makes use of computational domains that are generated separately. The two surface representations are first edited and smoothed independently. Some holes and cuts are filled intuitively on each skull representation without the assistance of a medical expert. Because 


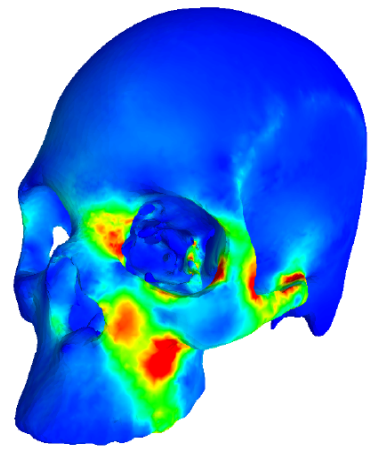

(a)

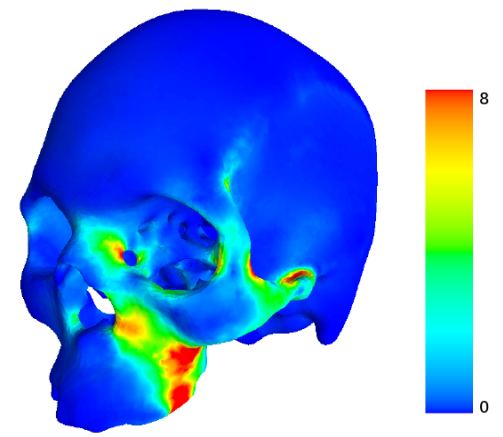

(b)

Figure 2: Von Mises stress contours for a molar bite for the range [0, 8] MPa on the (a) prognathic and (b) orthognathic skull shape using meshes independently generated on the edited surface representation.

of this, the skulls are not fully reconstructed. The initial difference in topology between the skull representations is therefore still largely maintained.

Tetrahedral finite element domains are created from the edited surface representations. Boundary conditions are set up for the prognathic and orthognathic representations with the force directions and magnitudes approximated and determined using the geometry itself and various similar studies as a guideline $[2,5,6]$. Isotropic linear elastic material properties are used with a Youngâs modulus of $E=16 \mathrm{GPa}$ and Poisson's ratio of $\nu=0.3$ [7-12]. The prognathic computational domain consists of 113104 nodes and 401455 elements, whereas the orthognathic computational domain is represented by 110645 nodes and 397354 elements.

Muscles included in the analysis are the temporalis, medial pterygoid, superficial masseter and deep head masseter. The fan-like temporalis muscle is approximated by seven segments. All muscle and muscle segment forces are applied at the nodes that approximately represent the muscle attachment location on the skull. These forces act in the approximate direction of attachment to the mandible, which was determined with the assistance of a medical expert. This means that the force directions are separately determined for the prognathic and orthognathic skull models. For a detailed outline of how the finite element model is set up, refer to [13].

Figure 2 shows the von Mises stress field results on the independently generated computational domains for a molar bite analysis. There is a perceived difference in stress field that could indeed be attributed to the difference in skull shape. The stress field comparison can only be carried out visually or directly compared at specific locations. The latter requires the consistent manual selection of corresponding points in each domain.

It is however possible that the difference in the reported stress field is not only attributed to a difference in skull shape. The comparison may also be sensitive to the relative difference in scale, asymmetry of the skulls analysed and topological variation. Topological variation between various skull geometries may reflect a true variation in areas such as the sinuses or possibly other internal membranes. It is also possible that varying degrees of decay in certain areas may result in topological inconsistencies where in fact there is none.

The digitally reconstructed prognathic and orthognathic skull shapes are visible in Figures 3 and 4. Side views are presented in Figure 3(b) and (d) to give an indication of the relative degree of prognathism, whereas the translucent figures presented in Figure 3(a) and (c) also allow the reader to view some of the differences in topology. In Figure 4, cuts made with a plane through 


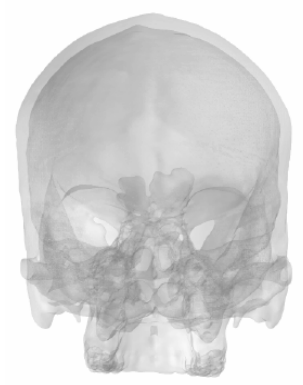

(a)

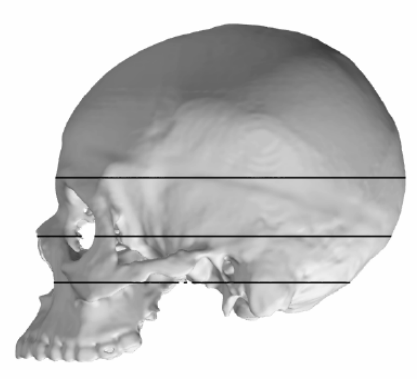

(b)

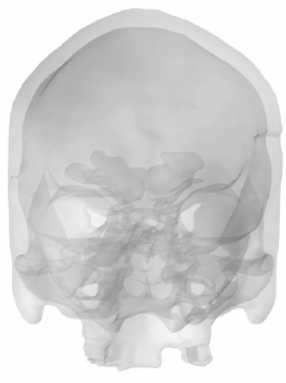

(c)

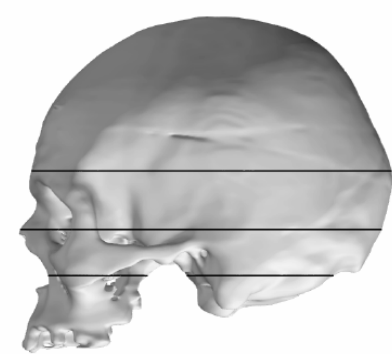

(d)

Figure 3: The surface mesh representations of the prognathic and orthognathic skull subjects. (a) and (c) are translucent frontal views of the surface representations to illustrate the variation in sinus size and shape. The side views in (b) and (d) contain lines used for the location of the cuts made in Figure 4.

the same approximate location of the two surfaces complement the views in Figure 3 in a further attempt to illustrate these differences.

In this study, it is required to test the variation in stress field because of a difference in shape only. With the geometries available for analysis, this could be partly achieved by editing the domains to reflect the exact same topology. In the remainder of this article, the use of non-rigid registration to obtain approximately symmetric and topologically consistent domain representations of each skull is illustrated. This is undertaken to inspect the variation in masticatory-induced stress field because of a difference in shape only.

\section{Non-rigid registration}

The elastic surface registration procedure of Moshfeghi et al. [14], as implemented and improved by Bryan et al. [15], is used as foundation. Bryan et al. [15] used the procedure to analyse the performance of orthopaedic implants while accounting for inter-patient variability with respect to bone quality and geometry. In [15], their procedure is used to create a three dimensional model of a femur that is ready for statistical analysis and FEA. Bryan et al. [15] registered 46 femur geometries and then statistically determined the modes of variation in both shape and material density.

For the work performed in this study, the generic skull shape used as the deformable surface mesh is created from the digital prognathic skull. The prognathic surface mesh is edited by filling the holes caused by decay and the incisions made postmortem to remove the brain. As in the independent mesh generation case, only small holes were filled intuitively without the assistance of a medical expert. The choice of the prognathic skull for the generic mesh generation is based mainly on the presence of dentition and less decay in the facial area, as compared with the orthognathic skull.

The available registration procedure, implemented from the work of Bryan et al. [15], was developed for objects of varying shape, but with identical topology. Using our implementation, misrepresentation and self intersection of the deformable surface occurs in areas where features and structures are present that are not common to both surfaces but are nevertheless allowed to be registered. A difference in topology, as well as overlapping and unmatched features, play a role in invalid registration and subsequent mesh deformation. 


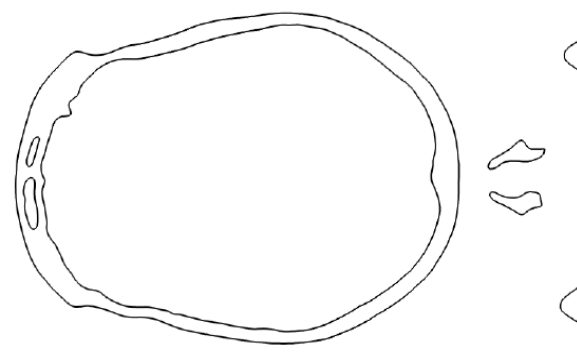

(a)

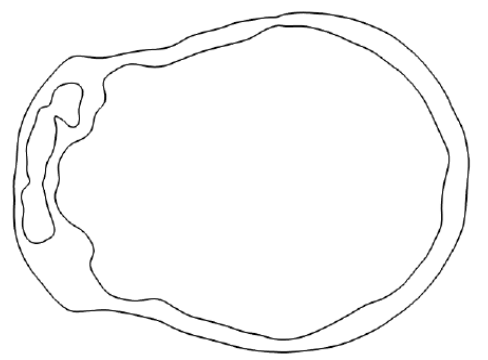

(d)

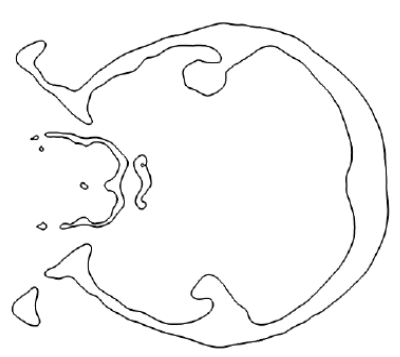

(b)

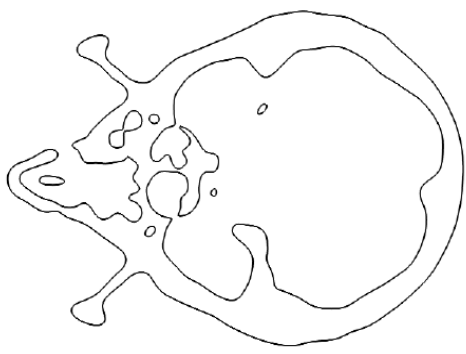

(e)

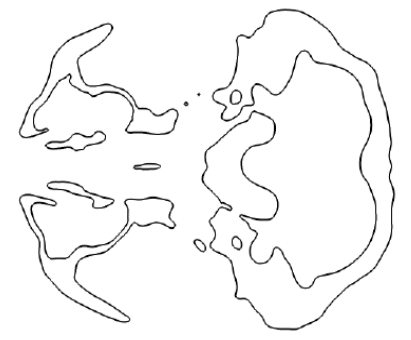

(c)
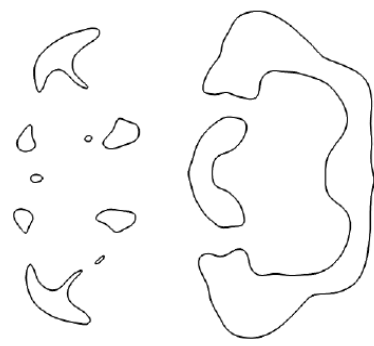

(f)

Figure 4: Cut planes made in an attempt to illustrate varying topology. (a)-(c) are intersections of the prognathic surface with the planes illustrated in Figure 3(b) with (d)-(f) the approximate equivalent on the orthognathic shape in Figure 3(d).

In Figure 5, full elastic surface registration performed on the orthognathic skull is visible. A large part of the target representation in Figure 5(c) seems to have undergone satisfactory registration to represent the orthognathic surface. Problems do however occur with surface noise, particularly visible internally and in the area of the sinuses.

These problems appear to arise from the topological inconsistencies when the generic and target shapes are compared. Because the registration procedure and the ideal stress field comparison require topological consistency, the topology of the generic representation is required to be fixed and consistent on all domains.

To use the available non-rigid registration procedure, a few constraints are required to ensure that topological inconsistencies do not play a role in inadequate or undesired registration. Alternatively, the target geometry could be edited to reflect the same topology as the generic shape, prior to non-rigid registration. The option to constrain the registration procedure rather than edit the target is chosen because a study using a larger sample of geometries should then require less user intervention.

The modified registration procedure, that is outlined in this section, consists of three distinguishable steps. Rigid registration is first performed to rotate, scale and translate the target shape in such a way that it is globally aligned to the deformable generic mesh. Curvature information is then used to extract feature information. Features on the generic and target shapes are compared and areas in the vicinity of mismatched features are classified as low confidence surface areas. Constrained nonrigid surface registration is subsequently performed using only higher confidence areas to deform the generic domain. The elastic surface registration procedure is presented in Appendix A of this article for easy reference. For further detail into the procedure, the reader is referred to the appendix of the article by Bryan et al. [15]. 


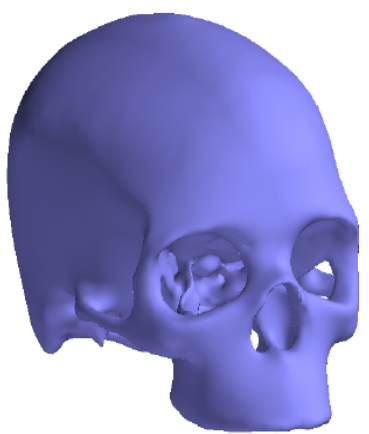

(a)

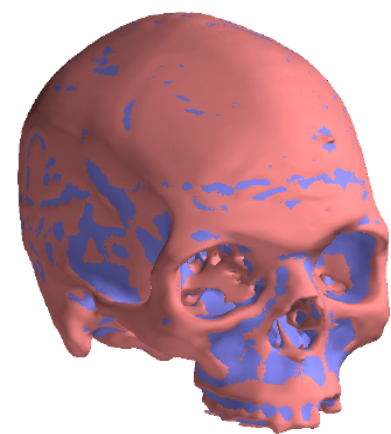

(b)

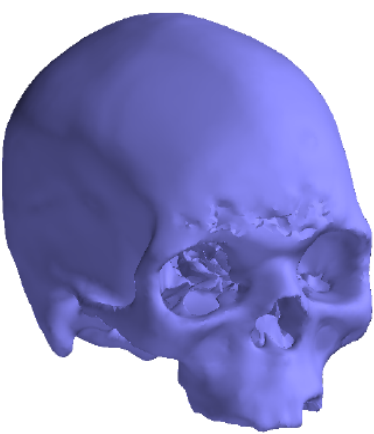

(c)

Figure 5: (a) Deformable model at iteration 0. (b) Deformable model at iteration 60 in blue overlayed on the pink orthognathic target surface. (c) Deformable model at iteration 60 . The same registration procedure parameters are used as Bryan et al. [15].

\subsection{Rigid registration}

Before deforming the generic skull shape into a representation of the target skull form, an affine registration is performed using an iterative closest point (ICP) method. Besl and McKay [16] approached the problem of obtaining a rigid transformation in point set registration in a least squares manner. Later versions of their method also attempt anisotropic scaling in addition to rotation and translation. One such method proposed by Du et al. [17] uses an iterative affine transformation implemented through the use of rotation, reflection and anisotropic scaling matrices with a translation.

The procedure of Du et al. [17] is used in this report to align a target shape with the deformable surface mesh prior to elastic surface matching and mesh morphing. Isotropic scaling is achieved with their procedure by including a modification that only allows a single scale variable during optimisation, instead of the three variables used in [17].

\subsection{The use of curvature information}

In this study, it is required to compare the stress in representative domains that have no difference in topology and scale. Because non-rigid registration is used to obtain the representative domains in this study, it would be preferable to only use the attributes associated with facial form to inform the deformation of the generic mesh. This is undertaken so that a noticed variation in stress field may be more likely as a result of the facial attributes, rather than the undesired possibility that a variation caused by topology or some other unmatched feature is reported.

To use the available non-rigid surface registration procedure, without the need to first edit a tar- get shape, registration constraints are determined using feature and curvature information. Feature lines are first extracted using the method proposed by Kim and Kim [18]. This method involves the approximation of an implicit surface at each point to extract curvature information. The principal curvatures and their derivatives are then used to connect possible feature points into lines.

Decayed areas were simply removed from the generic representation, therefore, the generic mesh used in this study is not representative of a fully reconstructed skull. Most of the features internal to the facial region of the generic representation are therefore artificial. The sinuses along with other artificial ridge and valley features of the internal facial area of the generic 


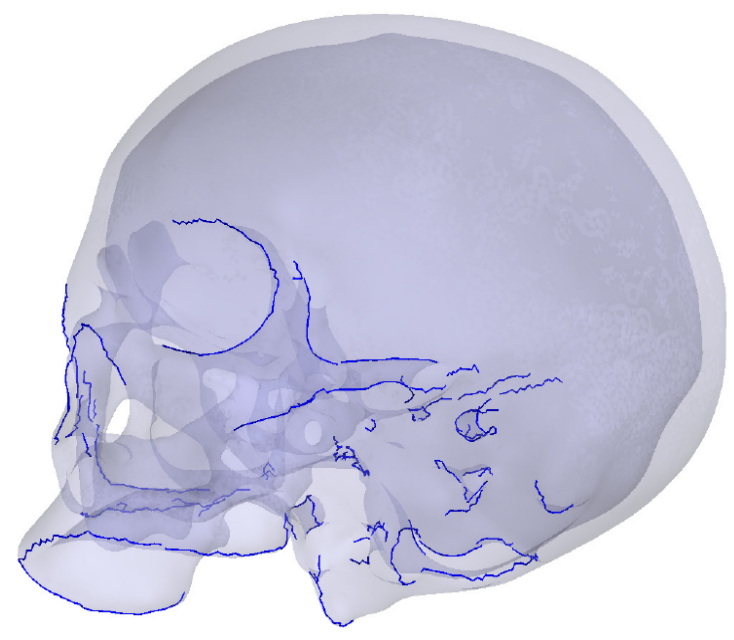

(a)

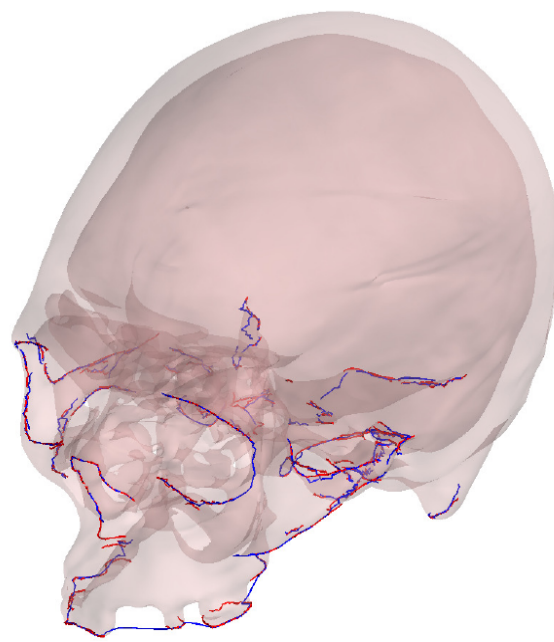

(b)

Figure 6: (a) User selected allowable feature lines on the model skull geometry. (b) The registered position of the model features and corresponding feature lines extracted on the orthognathic target geometry.

skull are removed from the allowable feature lines and surfaces in this study. This leaves mainly external features that allows the capture of overall shape of each skull, as is visible in Figure 6(a). Areas associated with these higher confidence features and salient areas on the generic shape are allowed to register during elastic surface matching and mesh morphing.

Using the allowable feature lines on the generic shape, feature line registration is performed to automatically detect corresponding higher confidence feature areas on a target prior to nonrigid surface registration. Feature line registration is performed with a procedure similar to that proposed by Subsol et al. [19]. In our implementation, the point correspondence and registration correction is carried out in the same way as explained by Subsol et al. [19].

In the procedure of Subsol et al. [19], three transformations are used during registration: a rigid transformation first aligns the sets of lines after which affine transformations retrieve the scalar differences between the sets. Spline transformations are finally used to determine and perform local deformation. In our procedure, the feature line registration is integrated into the non-rigid surface registration procedure. This means that the scale and orientation correction is carried out before the feature registration step. Using the elastic surface registration procedure outlined in Appendix A, it was decided that the local deformation would be performed iteratively, using the deformation calculation of the procedure instead of the spline transformation used by Subsol et al. [19].

Registration of the allowable feature lines on the generic skull to the orthognathic form is displayed in Figure 6(b). In this figure, the location of the deformed lines after registration to the orthognathic form is visible as the blue lines. The corresponding features registered on the target geometry is displayed using red lines. The unregistered features on the target surface are discarded and not depicted.

Registration to the high curvature surface areas associated with unmatched features are classified as untrusted and are automatically discarded. Points in high curvature areas are classified using the magnitudes of principal curvature information gathered during feature line 


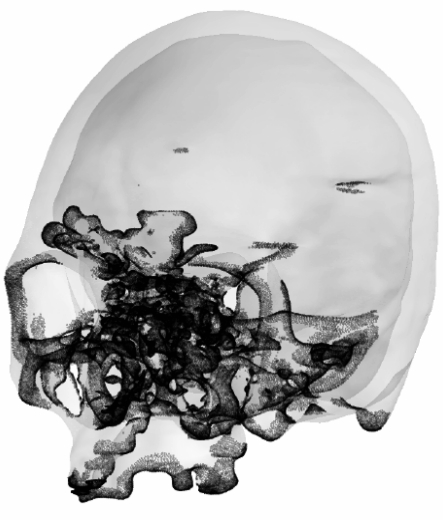

(a)

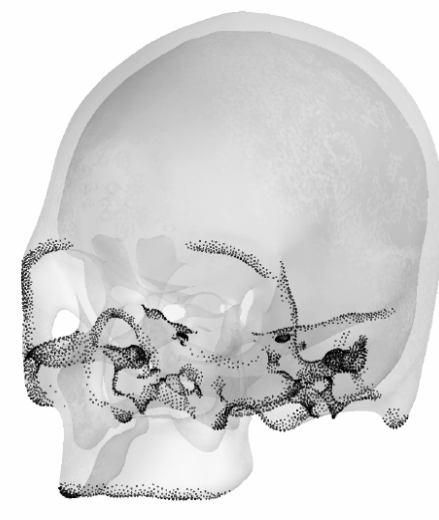

(b)

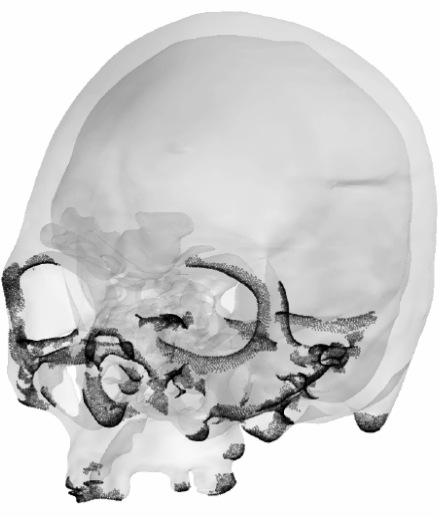

(c)

Figure 7: Mesh points on areas within the bounds of user selected curvature. (a) High curvature areas on the orthognathic skull target geometry. (b) High curvature areas corresponding to the user selected allowable feature lines seen in Figure 6(a). (c) The automatically selected allowable feature areas obtained after the feature registration on the orthognathic target geometry seen in Figure 6(b).

extraction.

Feature points satisfying the user specified curvature conditions on the orthognathic mesh are illustrated in Figure 7(a). The skulls have been scaled during the affine ICP procedure, so the relative scale difference in the curvature of corresponding features is assumed to be negligible. The length scale in this case is millimetres and curvatures are approximated at each vertex on the surface using neighbour vertices within a spherical radius of $5 \mathrm{~mm}$ to approximate the implicit surface. Only points with principal curvature values $\kappa_{\min } \leq-0.18 \mathrm{~mm}^{-1}$ or $\kappa_{\max } \geq 0.18 \mathrm{~mm}^{-1}$ are displayed and considered as part of high curvature feature areas in this study. Figure $7(\mathrm{~b})$ contains points with high curvature on the generic mesh that correspond to the user selected allowable feature lines displayed in Figure 6(a). After feature registration, the higher confidence feature areas on the target can be obtained by computing its relative distance to feature lines. If the closest feature line to a point is an unmatched line, then this point and the triangles that contain it are classified as part of an unmatched feature. Feature points on the orthognathic shape that are automatically classified as part of higher confidence features are visible in Figure 7(c).

The use of curvature information to inform and constrain the allowable surface registration areas is included in the procedure to reduce the amount of user intervention required. The selection of allowable features is performed once off on the generic mesh, without the requirement to edit the target in such a way as to obtain the same topology as the deformable generic shape. Should the procedure be implemented to retrieve approximate representations of a larger statistical sample of skull geometries, the inclusion of this step would translate into overall less user intervention.

\subsection{Computational domain preparation}

After excluding the areas where a mismatch in features could result in undesired registration, nonrigid surface registration is performed using the procedure of Bryan et al. [15]. The topology of the generic skull is now maintained although only deforming the domain to capture the overall shape and attributes of the prognathic and orthognathic shape. In their study, Bryan et al. [15] used a 


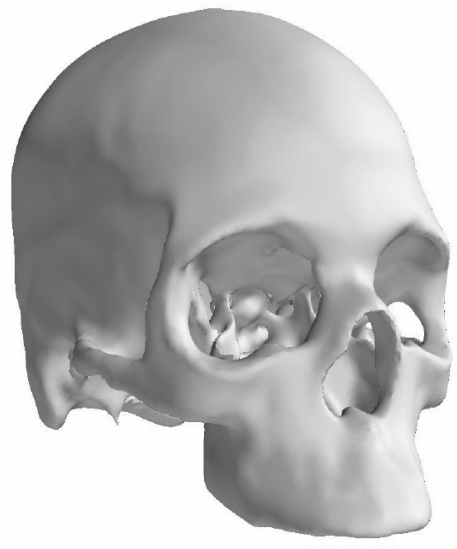

(a)

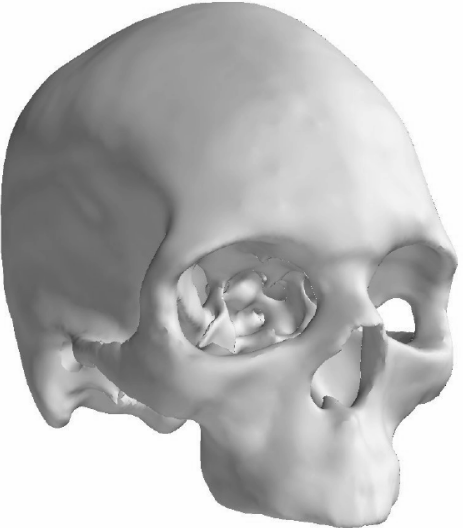

(b)

Figure 8: Approximate symmetric versions of the (a) prognathic and (b) orthognathic skull geometries.

nearest neighbour parameter $n=50$, the smoothing parameters $\gamma=2, \sigma_{0}=10$ and $f=1.0715$ and the maximum iterations $k_{\max }$ was set to 100 when registering the femur geometries. The same parameters are used here to register and deform the generic skull mesh.

For this study, a tetrahedral element mesh is generated on the generic triangular surface mesh. The tetrahedral mesh is deformed using the Gaussian weighting function implemented into the registration procedure. To make the elastic surface registration more robust, the deformation obtained as a result of the Gaussian function is smoothed using Taubin smoothing [20]. This smoothing acts as a low-pass filter to reduce unwanted high frequency deformation, making the inversion of elements less likely. A broad overview of the implemented Taubin smoothing procedure is presented in Appendix B.

Both prognathic and orthognathic skull geometries are registered and approximately represented by a deformed generic mesh. The target shapes are the original surfaces visible in Figure 3. The procedure for matching feature lines and determining lower confidence registrations is applied to both models, and the generic surface is deformed into target representations. The target geometries are left totally unedited with all the user specified constraints and restrictions only applied to the generic deformable mesh.

The generic model is deformed into a target shape as well as the reflected target shape. The average of the two deformed meshes is then used to create a symmetric version of the target. The near symmetric versions of both the orthognathic and prognathic skull geometries are illustrated in Figure 8. The stress fields in these symmetric representations are compared in the next section, to remove the effect of asymmetry. The cut planes shown in Figure 9 are of the symmetric domain representations. These cut planes are at the same approximate locations as the cut planes presented in Figure 4 of the original skull representations.

Because there is now a consistent mapping between the symmetric prognathic and orthognathic shapes, a mesognathic skull can now be created by averaging the nodal coordinates of these representations. A high quality tetrahedral mesh is then generated using this mesognathic skull surface. This is carried out so that the tetrahedral mesh would not result in a bias toward the prognathic skull during analysis. This domain is then deformed back into the prognathic and orthognathic skull representations using the known surface displacements. These known boundaries are used in linear elastic finite element analyses to determine the displacements of 


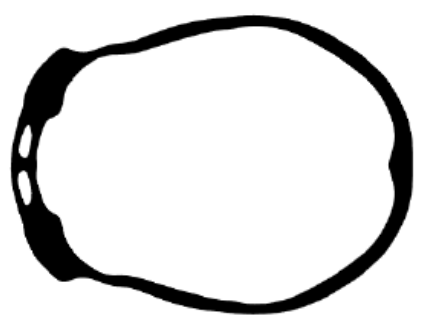

(a)

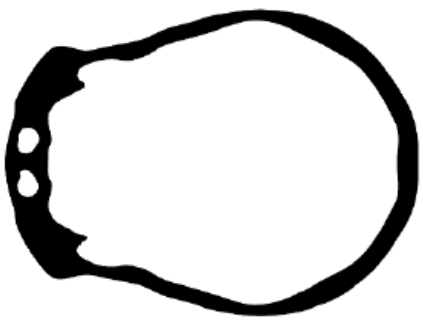

(d)

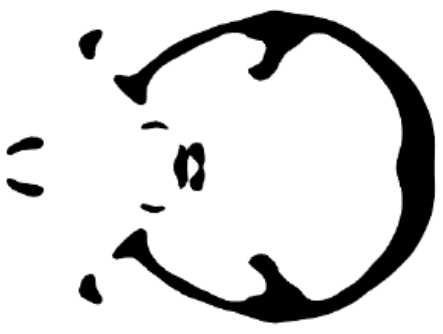

(b)

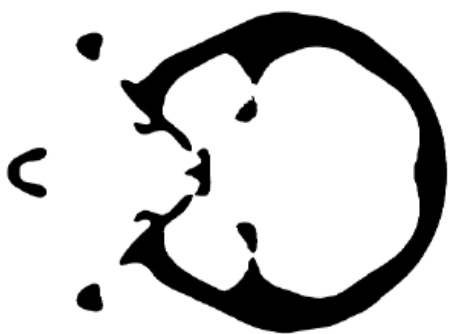

(e)

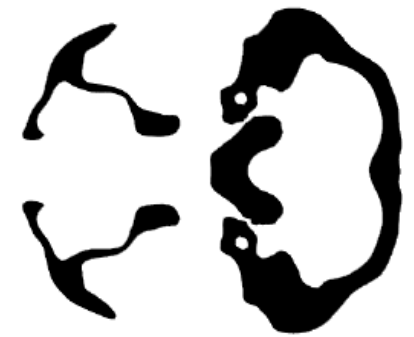

(c)
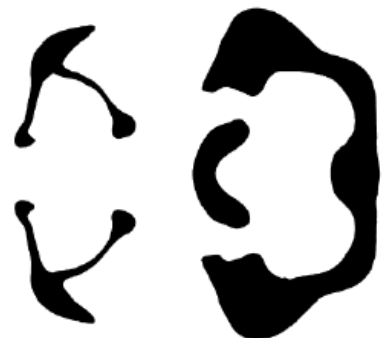

(f)

Figure 9: Cut planes made in an attempt to illustrate topology inherited from the generic domain on the symmetric representation of the prognathic and orthognathic skulls. The location of these cuts made on the symmetric representations correspond to those on the original surfaces presented in Figure 4.

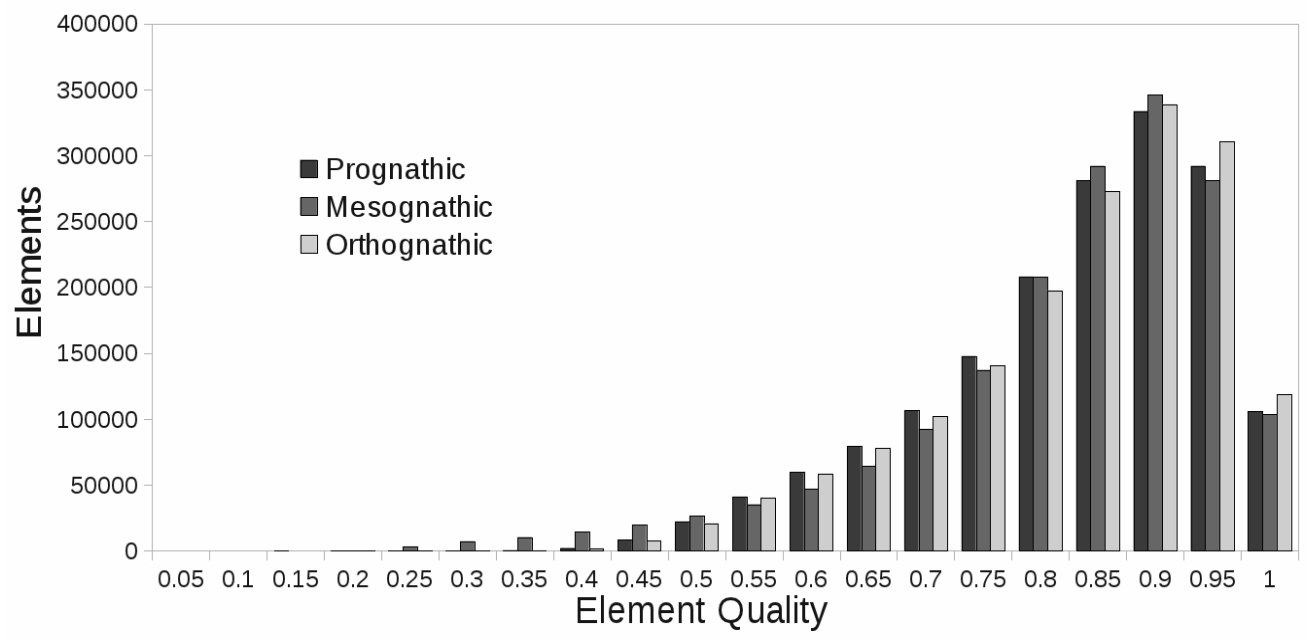

Figure 10: Element quality distribution of the prognathic, orthognathic and mesognathic skull tetrahedral meshes. Bin labels indicate the upper bound. 
internal nodes. The full nodal displacements are then applied to the mesognathic tetrahedral mesh to obtain the prognathic and orthognathic meshes.

The boundary coordinates of the tetrahedral mesh is constrained during mesh untangling and optimisation. This step is performed with the MESQUITE (MESQUITE Software, Inc, Austin, TX, USA) [21] mesh quality improvement toolkit. A histogram illustrating the element quality distribution of the prognathic, mesognathic and orthognathic tetrahedral meshes after optimisation is visible in Figure 10. A brief overview of MESQUITE and further details on the element quality metric is given in Appendix C. From the element quality distribution in Figure 10 there seems to be no bias toward either prognathic or orthognathic mesh representation.

\section{Comparing masticatory induced stress}

The three tetrahedral meshes representing prognathic, mesognathic and orthognathic form are analysed for an applied molar bite force. The nodes, where boundary conditions are applied, are the exact same nodes for all three domain representations. All three meshes consist of 290 569 nodes and 1687791 elements. A finer mesh is used than those independently analysed. This is done in the hope that the elements are more likely to maintain good quality during mesh deformation. The boundary conditions and material properties are handled in the same way as discussed in Section 2. The direction of the muscle forces for the mesognathic case is obtained by interpolating between the known directions in the prognathic and orthognathic cases. If the models included mandibles, muscle force direction would be determined completely by using the node numbers that represent the attachment location on the mandible for that muscle.

In Figure 11, the Von Mises stresses are given with all stresses higher than $8 \mathrm{MPa}$ simply shown in red. The maximum resulting Von Mises stress for the analyses are 18.56 MPa for the prognathic skull form, $15.334 \mathrm{MPa}$ on the mesognathic skull form and $17.589 \mathrm{MPa}$ for the orthognathic skull form respectively.

If the requirement is simply to analyse the appropriate domains obtained by non-rigid registration, these results may be compared in the same way as the independently meshed results in Figure 2. Figures 11(a) and (c) compare well with the results displayed in Figures 2(a) and (b). The difference in stress field visible between the prognathic and orthognathic shapes in Figure 11 is no longer affected by asymmetry and topological variation, whereas the results displayed for comparison in Figure 2 do implicitly contain these effects. Because a target geometry is isotropically scaled to the generic mesh in the ICP alignment, it may also be assumed that the effect of scale on the variation in stress field is negligible.

To illustrate the unintended benefit of obtaining appropriate domain representations by nonrigid registration, the results of the FEA are manipulated using this one to one domain correspondence already available. In Figure 12, resulting von Mises stress fields in the prognathic and orthognathic forms are compared. Here, the von Mises stresses in the orthognathic skull domain and prognathic skull domain are displayed at the approximate location of those elements on the mesognathic domain representation.

The results are compared in Figure 12(c) by taking the difference in von Mises stress. The difference in the Cauchy stress tensor for instance is not an accurate representation of the actual difference in stress. Stress tensors are recovered from a FEA on different geometries with the same mesh topology. For each mesh, the same element is likely to have a different global orientation. A proper interpolation scheme is therefore required or one may compare invariants of the stress tensor such as the eigenvalues (principal stresses). Von Mises stress is used in this example because it is also rotationally invariant. 


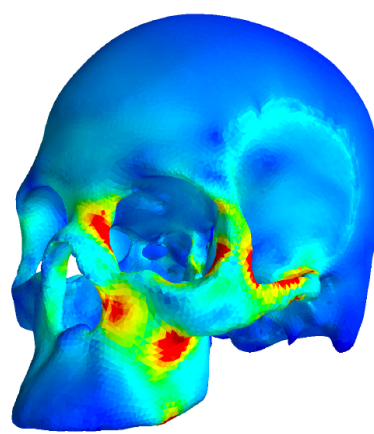

(a)

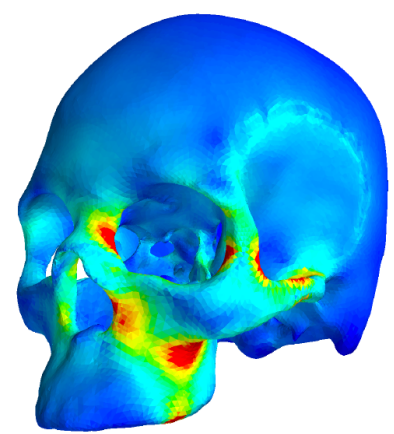

(b)

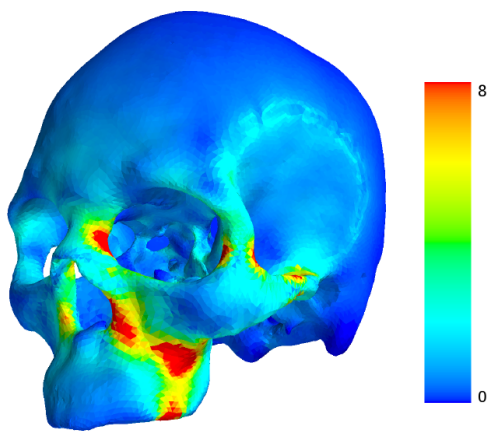

(c)

Figure 11: Von Mises stress contours for a molar bite for the range [0,8] MPa on the (a) prognathic, (b) mesognathic and (c) orthognathic skull shape.

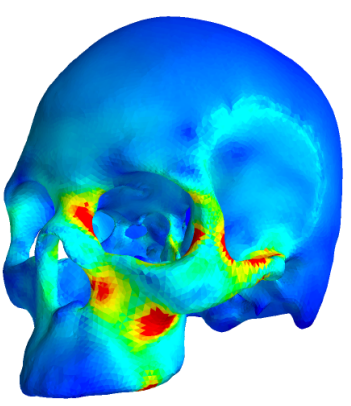

(a)

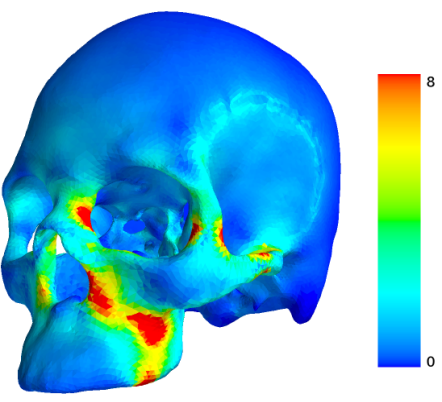

(b)

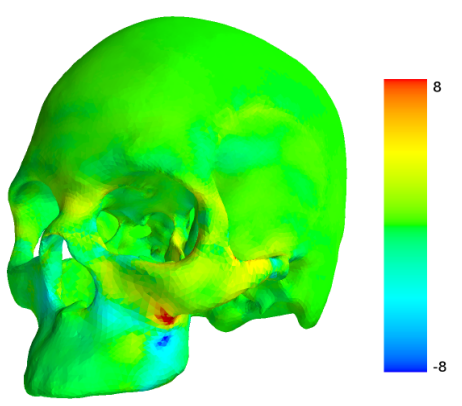

(c)

Figure 12: Von Mises stress contours for a molar bite for the range $[0,8] \mathrm{MPa}$ of the (a) prognathic and (b) orthognathic skull shape plotted on the mesognathic skull shape. (c) The contours of difference in Von Mises stress between the analysed shapes $\left(\sigma_{\text {prognathic }}^{\mathrm{vM}}-\sigma_{\text {orthognathic }}^{\mathrm{vM}}\right)$ for the range $[-8,8] \mathrm{MPa}$ on the mesognathic skull shape. 
From the variation in masticatory induced stress field presented in Figure 12(c), it would appear from this analysis that there is a higher stress in the zygomatic arch and bridge of the nose in the prognathic skull form, while the orthognathic form has a higher stress concentration in the maxilla or upper mandible. This report does not however aim to draw conclusions between prognathism and stress. This report merely inspects the use of non-rigid registration in such a study. Before a claim can be made on the effect of prognathism and stress, a detailed study is required. This detailed study would require a larger number of skulls, modelled with higher detail and combined with a statistical analysis. A study on prognathismâs effect on masticatory induced stress would also require greater input from an anthropologist or some other interested medical professional to set up models and draw conclusions.

\subsection{The effect of mapping uncertainty}

If the non-rigid registration procedure is merely used as a means to extract an appropriate domain representation, a possible uncertainty in the non-rigid map between domains would present no concern. In this study however, the mapping is also used to make objective and direct comparisons. It would be undesirable to assign significance to a specific variation in stress or some other quantity owing to some uncertainty in the accuracy of the mapping. Note that this mapping uncertainty is inherently part of this type of problem and not caused by some limitation of the chosen elastic registration procedure. When mapping one skull form to another, an exact solution does not exist, and any registration procedure merely attempts to provide some realistic mapping.

Various meshes representing the orthognathic skull geometry are created in an attempt to illustrate and quantify the effect of registration uncertainty and discretisation on the FEA results and subsequent comparison. The non-rigid registration procedure used in this specific study requires user defined registration and smoothing parameters. Two additional meshes are generated using the symmetric orthognathic skull as the target during non-rigid registration. The registration is performed using two sets of smoothing parameters:

- $\gamma=2, \sigma_{0}=10$ and $f=1.0715$ and

- $\gamma=2, \sigma_{0}=20$ and $f=1.0715$.

The original surface mesh representing the symmetric orthognathic skull shape along with the result obtained from the two additional registrations are visible in Figure 13. Figure 13(a) shows that the three meshes represent the same geometry, whereas the detail of Figure 13(b) shows that there is not an exact solution.

The tetrahedral mesh, generated on the mesognathic surface mesh is deformed using the displacement of boundary nodes and again optimised using MESQUITE [21].

When referring to the prognathic mesh, the symbol P1 is used. $\mathrm{O} 1$ is the original orthognathic mesh with $\mathrm{O} 2$ and $\mathrm{O} 3$ as the two additional meshes generated to represent the orthognathic skull form. The von Mises stresses of the results of a molar bite simulation on the three orthognathic meshes are given in Figure 14. Figure 14(a) is a side view of the original analysis also displayed in Figure 11(c). Along with this result, the von Mises stress on the other two meshes representing the orthognathic form are displayed in Figures 14(b) and (c). From this figure, it is visible that essentially the same analysis is performed.

The results of the three orthognathic meshes are compared with the result on the prognathic mesh in the same way as in Figure 12(c). With a slightly different mapping between the prog- 


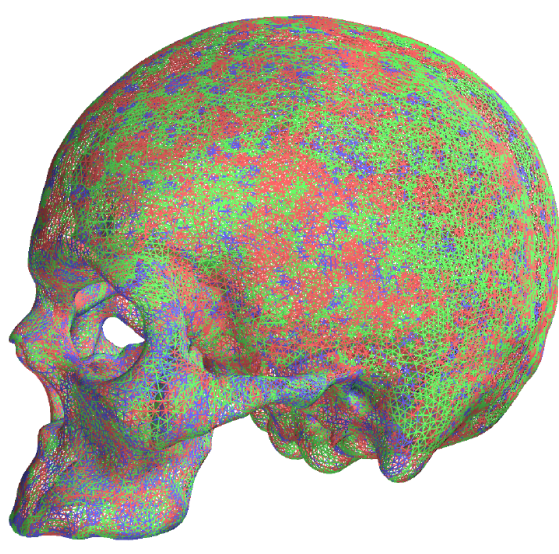

(a)

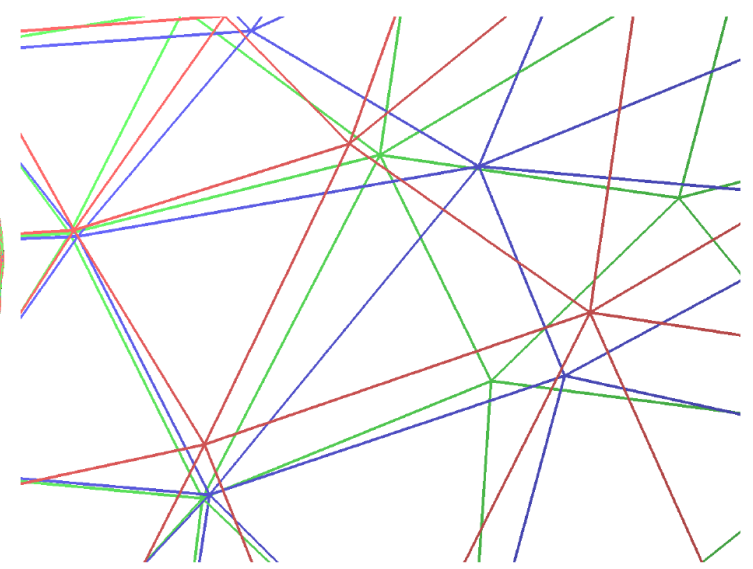

(b)

Figure 13: (a) Three different meshes representing the same orthognathic skull geometry. (b) Detail of the meshes in (a) illustrates non-uniqueness of nodal coordinate positions.

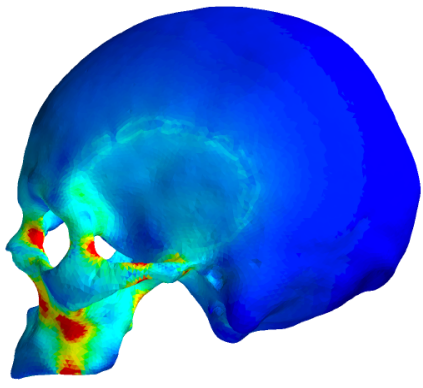

(a)

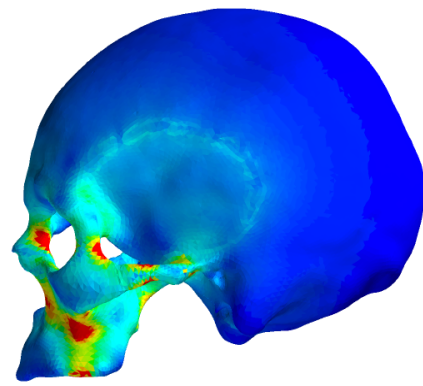

(b)

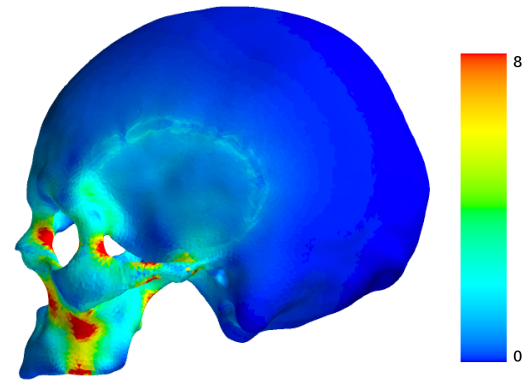

(c)

Figure 14: Analysis of molar bite induced stress on the three different orthognathic skull representations. 


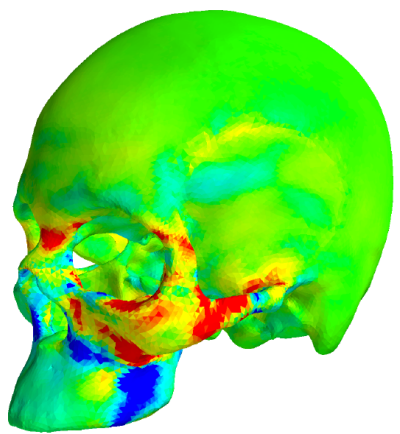

(a)

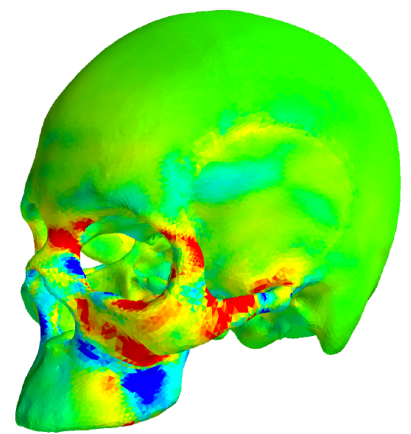

(b)

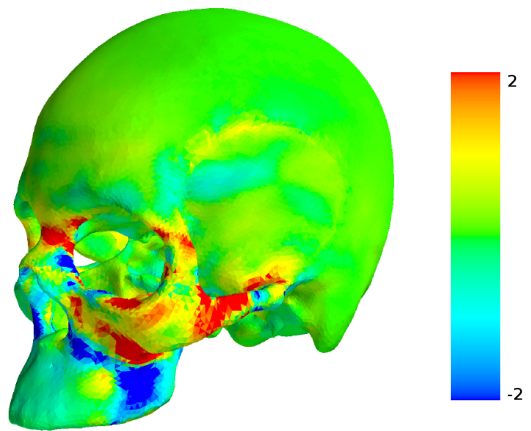

(c)

Figure 15: The difference in Von Mises stress for various combinations of the prognathic and orthognathic molar bite analyses. Contours are given for the range $[-2,2] \mathrm{MPa}$. The calculated range is (a) $[-7.978,12.839] \mathrm{MPa}$ for $\left(\sigma_{\mathrm{P} 1}^{\mathrm{vM}}-\sigma_{\mathrm{O} 1}^{\mathrm{vM}}\right)$, (b) $[-7.171,12.332] \mathrm{MPa}$ for $\left(\sigma_{\mathrm{P} 1}^{\mathrm{vM}}-\sigma_{\mathrm{O} 2}^{\mathrm{vM}}\right)$ and $(\mathrm{c})[-7.584,12.232] \mathrm{MPa}$ for $\left(\sigma_{\mathrm{P} 1}^{\mathrm{vM}}-\sigma_{\mathrm{O} 3}^{\mathrm{vM}}\right)$ respectively.

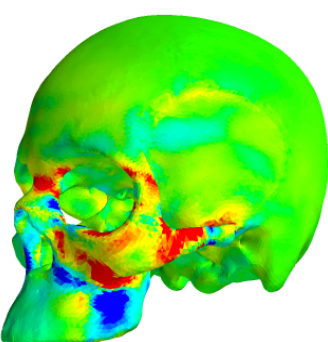

(a)

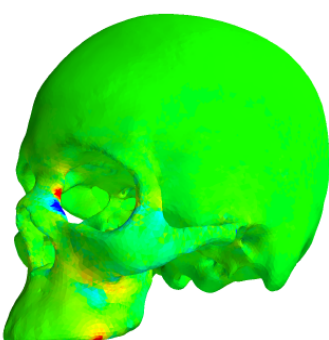

(b)

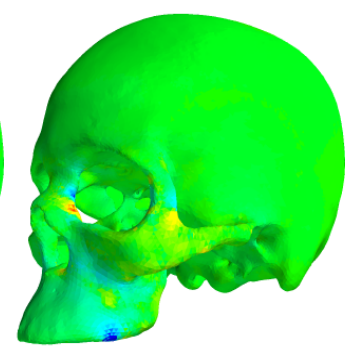

(c)

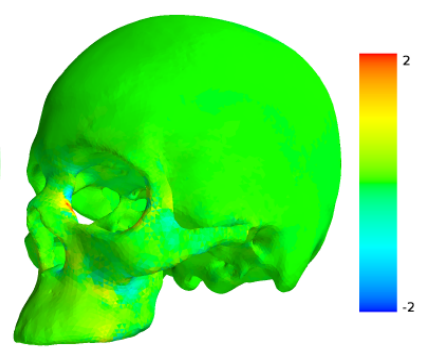

(d)

Figure 16: (a) Average of the three comparisons. (b)-(d) Deviation of the three direct comparisons from the average.

nathic and each orthognathic shape, the difference in von Mises stress field varies as depicted in Figure 15.

Figure 15(a) is the same results as illustrated in Figure 12(c) but shows contours for $\sigma_{\mathrm{P} 1}^{\mathrm{vM}}-\sigma_{\mathrm{O} 1}^{\mathrm{vM}}$ in the range $[-2,2] \mathrm{MPa}$. Figures $15(\mathrm{a})$, (b) and (c) are generated by comparing the same prognathic mesh result $\left(\sigma_{\mathrm{P} 1}^{\mathrm{vM}}\right)$ with the three different orthognathic mesh results $\left(\sigma_{\mathrm{O} 1}^{\mathrm{vM}}, \sigma_{\mathrm{O} 2}^{\mathrm{vM}}\right.$ and $\left.\sigma_{\mathrm{O} 3}^{\mathrm{vM}}\right)$.

Figure 15 only shows contours of the difference in Von Mises stress for the range $[-2,2] \mathrm{MPa}$, with the true range interval for each comparison given in the figure caption. Slight variation is noted in the difference in Von Mises stress between the prognathic and orthognathic shape when comparing these results.

In this case, although a slight variation in stress field pattern is visible in Figure 15, the overall difference in stress field between the two skull forms analysed may likely be attributed to the difference in form and not to the uniqueness of the mapping used between them.

The variation in difference in von Mises stress is inspected in Figure 16. The average of the results in Figure 15 is used as a baseline, and it is depicted in Figure 16(a). Figures 16(b) to (d) are the deviations of the original comparisons from the baseline.

From these results, it seems that although the analyses on the different orthognathic represen- 
tations are similar, the variation in how one geometry is mapped to the other does affect the comparison. In this particular study, the effect of mapping uncertainty only contributes marginally to the stress variation result. The use of non-rigid registration to obtain computational domain representations allows the opportunity to at least approximately evaluate dissimilarity in stress field because of shape in this objective and direct manner.

\section{Discussion}

From the results presented in Section 3, it seems that a study on the effect of only shape on stress results performed with the use of non-rigid registration is feasible. The use of numerical techniques such as the one discussed in this article allows the isolation of shape from other variations that could affect the results. An added benefit revealed through the use of nonrigid registration to acquire appropriate domain representation is the possibility of direct and objective comparison and manipulation. If direct comparison or statistics is carried out using the consistent domain representations, it is however important to inspect and quantify the effect of the direct mapping uncertainty.

Deforming one human skull domain or a representative human skull domain to better represent another has no true equivalent in nature and cannot be determined or verified experimentally. In a study of this type, where comparison is undertaken using non-rigid registration, it is only possible to compare distinguishable landmark locations and force these to map exactly. The challenge lies in obtaining an accurate map between other discretised and specifically featureless areas where there is no known exact solution to the mapping. For this reason, a simple quantification of uncertainty in the mapping is also presented along with the direct comparison.

The various direct comparisons presented illustrates that the difference in stress field displayed in this manner is slightly affected by the non-rigid mapping. The effect of a difference in shape is however orders of magnitude bigger than the effect of mapping uncertainty in the results presented. The use of non-rigid registration as a direct and objective comparison technique is a viable option to at least determine and visualise the approximate areas where a difference in stress field may be observed.

In further work, a more detailed generic skull model should be reconstructed. Conclusions on the effect of skull shape on stress field should also be drawn with the assistance of a medical professional. If a larger statistical sample of skull geometries are used and analysed with a consistent mapping between them, principal components of shape or modes of deformation and stress could be extracted with relative confidence. The difference in stress or displacement from the mean stress and deformation caused by some mode of variation could then be better approximated and illustrated.

\section{Appendix A: Non-rigid surface registration}

Two meshes are taken as an input with $\mathcal{M}$ the generic and $\mathcal{P}$ the target surface. These two surface meshes consist of point data and connectivity lists defining the triangle patches that defines the surface. The generic surface is deformed iteratively to better represent the target without affecting connectivity. This is done as follows:

- Registration inputs are specified. These include the target mesh $\mathcal{P}$ and base mesh $\mathcal{M}$. Other user specified parameters are a nearest neighbour parameter $n$, smoothing parameters $\gamma, \sigma_{0}$ and $f$, maximum number of iterations $k_{\max }$ and stopping criteria tolerance 
- Rigid registration is performed to align the target geometry to the generic mesh using an ICP procedure.

- The iteration counter and deformable surface is initialised so that $k=1$ and $\mathcal{W}^{0}=\mathcal{M}$. Registration is performed while $k \leq k_{\max }$ or until the convergence criteria is met:

- Four $k-d$ tree representations are constructed. This is done for the centroids of surface triangulations and nodal coordinates of both $\mathcal{W}^{k-1}$ and $\mathcal{P}$.

- For each node $\mathbf{w}_{j}$ in $\mathcal{W}^{k-1}, j \in\left\{1,2, \ldots, N_{m}\right\}$, a registration to the target surface is determined. The $n$ nearest target triangles to $\mathbf{w}_{j}$ and registration points on these closest triangles are determined. The point $\mathbf{r}_{w_{j}}$ is produced by drawing a line from the point $\mathbf{w}_{j}$ perpendicular to the plane of each registered triangle. A distance measure is then assigned to the triangles. If the registered point $\mathbf{r}_{w_{j}}$ lies inside a triangle, the distance measure is computed as $\left|\mathbf{r}_{w_{j}}-\mathbf{w}_{j}\right|$. Alternatively, if the point lies outside a triangle the distance measure is computed as $\left|\mathbf{r}_{w_{j}}-\mathbf{w}_{j}\right|+\left|\mathbf{p}_{w_{j}}-\mathbf{r}_{w_{j}}\right|$, where $\mathbf{p}_{w_{j}}$ is the closest vertex of that triangle to point $\mathbf{w}_{j}$. Inspecting all the distance measures, the closest triangle to point $\mathbf{w}_{j}$ and the registration associated with it is obtained. If the point $\mathbf{r}_{w_{j}}$ lies inside the closest triangle, the displacement is calculated as $\mathbf{d}_{w_{j}}=$ $\mathbf{r}_{w_{j}}-\mathbf{w}_{j}$. Alternatively, if the point lies outside the triangle the displacement is calculated as $\mathbf{d}_{w_{j}}=\mathbf{p}_{w_{j}}-\mathbf{w}_{j}$.

- This registration procedure is then done again for $\mathcal{P}$ onto $\mathcal{W}^{k-1}$. For each node $\mathbf{p}_{i}$ in $\mathcal{P}, i \in\left\{1,2, \ldots, N_{p}\right\}$, a displacement to the deformable surface is determined as $\mathbf{d}_{p_{i}}=\mathbf{r}_{p_{i}}-\mathbf{p}_{i}$ if the point $\mathbf{r}_{p_{i}}$ lies inside the closest triangle or $\mathbf{d}_{p_{i}}=\mathbf{m}_{p_{i}}-\mathbf{p}_{i}$ if the point $\mathbf{r}_{p_{i}}$ lies outside the closest triangle.

- Having registered $\mathcal{W}^{k-1}$ onto $\mathcal{P}$ and $\mathcal{P}$ onto $\mathcal{W}^{k-1}$, a smooth displacement field is computed for a point $\mathbf{x}$ as

$$
S^{k-1}(\mathbf{x})=\frac{1}{\gamma}\left[\frac{\sum_{j=1}^{N_{m}} G\left(\left\|\mathbf{x}-\mathbf{w}_{j}\right\|\right) \mathbf{d}_{w_{j}}}{\sum_{j=1}^{N_{m}} G\left(\left\|\mathbf{x}-\mathbf{w}_{j}\right\|\right)}-\frac{\sum_{j=1}^{N_{m}} G\left(\left\|\mathbf{x}-\mathbf{r}_{p_{i}}\right\|\right) \mathbf{d}_{p_{i}}}{\sum_{j=1}^{N_{m}} G\left(\left\|\mathbf{x}-\mathbf{r}_{p_{i}}\right\|\right)}\right] .
$$

In Equation (2), $G(d)$ is the Gaussian weighting function suggested by Moshfeghi [14]:

$$
G(d)=e^{-d / \sigma_{k}^{2}}
$$

where $d$ is a positive scalar distance measure. The smoothing parameter $\sigma_{k}$ is decreased at each iteration allowing for more compact support using the update $\sigma_{k}=$ $\sigma_{0} f^{-k}$ with $1 \leq f \leq 2$.

- The deformable surface is updated as

$$
\mathcal{W}_{j}^{k}=\mathcal{W}_{j}^{k-1}+S^{k-1}\left(\mathbf{w}_{j}\right) .
$$

- To prevent mesh folding, Bryan et al. [15] performed a set number of improved Laplacian smoothing iterations to the deformable mesh $\mathcal{W}_{j}^{k}$ before performing another registration iteration. In our work, ten Taubin smoothing [20] operations are performed every five elastic surface registration iterations. This type of smoothing is chosen for it's ability to reduce high frequency surface noise without the high loss of volume associated with Laplacian smoothing. 
- Convergence is determined on the average total deformation as applied at the current iteration. The solution is terminated if

$$
\varepsilon=\frac{1}{N_{m}} \sum_{j=1}^{N_{m}}\left|S^{k-1}\left(\mathbf{w}_{j}\right)\right| \leq \varepsilon_{T} .
$$

The updated nodal coordinates of the generic mesh is returned after reaching a stopping criterion. This stopping criterion is either the satisfaction of Equation (5), the maximum number of iterations reached or no further improvement on the error value $\varepsilon$.

\section{Appendix B: Taubin smoothing}

Taubin smoothing extends signal processing to signals defined on polyhedral surfaces of arbitrary topology, reducing the problem of surface smoothing to a low-pass filter problem [20]. The low frequency content of the signal is regarded as subjacent data while the high frequency content is seen as noise. In this smoothing procedure, Fourier analysis is extended to signals defined on polyhedral surfaces based on the observation that the classical Fourier transform decomposes a signal into a linear combination of the eigenvectors of the Laplacian operator. A new operator is defined to take the place of the Laplacian.

Consider a polygonal curve or surface represented as a list of consecutive vertices $\mathbf{x}_{i}, i \in$ $\{1,2, \ldots, N\}$ with the neighbouring vertices of each point $i$ given in the connectivity information $i^{\star}$. If a discrete surface signal is a function $\phi=\left\{\phi_{1}, \phi_{2}, \ldots, \phi_{N}\right\}$, the discrete Laplacian of the discrete surface signal by weighted averages over the neighbourhoods may be defined as

$$
L_{i}=\frac{1}{\sum_{j \in i^{\star}} \omega_{i j}} \sum_{j \in i^{\star}} \omega_{i j}\left(\phi_{j}-\phi_{i}\right) .
$$

The weights $\omega_{i j}$ are positive numbers, with the simplest choice $\omega_{i j}=1$. Taubin [20] proposed the use of alternate scale factors of opposite sign $\lambda$ and $\mu$ with $\lambda+\mu<0,0<\lambda<1$ such that a vertex coordinate update may be determined iteratively by

$$
\mathbf{x}_{i} \leftarrow \mathbf{x}_{i}+\lambda L_{i} \mathbf{x}_{i} \leftarrow \mathbf{x}_{i}+\mu L_{i}
$$

The implementation in this article used $\lambda=0.5$ and $\mu=-0.53$.

\section{Appendix C: Usable mesh generation}

The MESQUITE mesh quality improvement toolbox is used to untangle and optimise the tetrahedral meshes in this article. The toolbox consists of libraries and header files providing an array of quality metrics, objective function assembly templates and quality improvement algorithms written in $\mathrm{C}++$.

The mean ratio quality metric is used for element shape optimisation in this article and may be constructed analytically [22]. If $\mathbf{Q}$ is a $n \times n$ matrix with $\operatorname{det}(\mathbf{Q})>0$, the mean ratio of $\mathbf{Q}$ is the scalar

$$
q_{\mu}=\frac{n \operatorname{det}(\mathbf{Q})^{2 / n}}{\|\mathbf{Q}\|_{F}^{2}}
$$


with $\|\mathbf{Q}\|_{F}^{2}$ the Frobenius norm of the matrix. In this article, tetrahedral elements are used and so the quality is evaluated with respect to an equilateral tetrahedron with all of it's edges unit length. If $\mathbf{J}$ is the Jacobian matrix referenced to node $\mathbf{x}_{0}$ on a particular element of concern and $\mathbf{W}$ is the Jacobian matrix referenced to node $\mathbf{x}_{0}$ of the equilateral tetrahedron, $\mathbf{Q}=\mathbf{J} \mathbf{W}^{-1}$ is the $3 \times 3$ Jacobian matrix of the affine transformation that maps the element to the reference shape. This quality metric is used in the article and is the metric used to show the quality of the meshes in Figure 10.

\section{Acknowledgements}

We acknowledge the assistance of M.L. Mac Kay and Prof. M. Steyn from the Anthropology Department of the University of Pretoria and thank the Department of Anatomy of the University of Pretoria for making their skull collection available for this study. We also acknowledge Labuschagne and partners at the Little Company of Mary Hospital for producing the CT scans used in reconstructing digital surface representations of the skulls.

\section{References}

[1] Panagiotopoulo O. Finite element analysis (FEA): applying an engineering method to functional morphology in anthropology and human biology. Annals of Human Biology 2009; 36(5):609-623.

[2] Richmond B, Wright B, Grosse I, Dechow P, Callum F, Spencer M, Strait D. Finite element analysis in functional morphology. The Anatomical Record Part A 2005; 283A:259-274.

[3] Cook R, Malkus D, Plesha M, Witt R. Concepts and applications of Finite Element Analysis. John Wiley \& Sons: New York, 2002.

[4] Robertson J. More on skeletal analysis and the race concept. Current Anthropology 1979; 20(3):617.

[5] Blanksma N, van Eijden T. Electromographic heterogeneity in the human temporalis muscle. Journal of Dental Research 1990; 69:1686-1690.

[6] Strait D, Richmond B, Spencer M, Callum F, Dechow P, Wood B. Masticatory biometrics and its relevance to early hominid phylogeny: An examination of palatal thickness using finite-element analysis. Journal of Human Evolution 2007; 52:585-599.

[7] Boryor A, Geiger M, Hohmann A, Wunderlich A, Sander C, Sander F, Sander F. Stress distribution and displacement analysis during an intermaxillary disjunction - a three-dimensional FEM study of a human skull. Journal of Biomechanics 2008; 41:376-382.

[8] Camacho D, Hopper R, Lin G, Myers B. An improved method for finite element mesh generation of geometrically complex structures with application to the skullbase. Journal of Biomechanics 1997; 30:1067-1070.

[9] Groning F, Liu J, Fagan M, O'Higgins P. Validating a voxel based finite element model of a human mandible using digital speckle patter interferometry. Journal of Biomechanics 2009; 42:1224-1229. 
[10] Ichim I, Sawin M, Kieser J. Mandibular biomechanics and development of the human chin. Journal of Dental Research 2006; 85:638-642.

[11] Kupczik K, Dobson C, Fagan M, Crompton R, Oxnard C, O’Higgins P. Assessing mechanical function of the zygomatic region in macaques: validation and sensitivity testing of finite element models. Journal of Anatomy 2007; 210:41-53.

[12] Tanne K, Miyasaka J, Yamagata Y, Sachdeva R, Tsutsumi S. Three-dimensional model of the human craniofacial skeleton: method and preliminary results using finite element analysis. Journal of Biomedical Engineering 1988; 10:246-252.

[13] Jansen van Rensburg G. Selective feature preserved elastic surface registration in complex geometric morphology. Master's Thesis, University of Pretoria, South Africa 2011. (Available from: http://upetd.up.ac.za/UPeTD.htm).

[14] Moshfeghi M, Ranganath S, Nawyn K. Three-dimensional elastic matching of volumes. IEEE Transactions on Image Processing 1994; 3(2):128-138.

[15] Bryan R, Mohan P, Hopkins A, Galloway F, Taylor M, Nair P. Statistical modelling of the whole human femur incorporating geometric and material properties. Mechanical Engineering and Physics 2010; 32:57-65.

[16] Besl P, McKay H. A method for registration of 3-D shapes. IEEE Transactions on Pattern Analysis and Machine Intelligence 1992; 14:239-256.

[17] Du S, Zheng N, Ying S, Liu J. Affine iterative closest point algorithm for point sets registration. Pattern Recognition Letters 2010; 31:791-799.

[18] Kim S, Kim C. Finding ridges and valleys in a discrete surface using a modified MLS approximation. Computer Aided Design 2006; 38:173-180.

[19] Subsol G, Thirion J, Ayache N. A general scheme for automatically building 3D morphometric anatomical atlasses: application to a skull atlas. Medical Image Analysis 1998; 2:37-60.

[20] Taubin G. A signal processing approach to fair surface design. Proceedings of the 22nd Annual Conference on Computer Graphics and Interactive Techniques, Los Angeles, 1995; $351-358$.

[21] Mesquite. (Available from: http://www.cs.sandia.gov/optimization/knupp/Mesquite.html).

[22] Escobar J, Rodrigues E, Montenegro R, Montero G, Gonzalez-Yuste J. Simultaneous untangling and smoothing of tetrahedral meshes. Computer Methods in Applied Mechanics and Engineering 2003; 192:2775-2787. 